\title{
Wall pressure beneath a transitional hypersonic boundary layer over an inclined straight circular cone
}

\author{
Siwei Dong ${ }^{1,2}$, Jianqiang Chen ${ }^{1,2}$, Xianxu Yuan ${ }^{1,2^{*}}\left(\mathbb{D}, X_{i} C^{1}{ }^{1,2}\right.$ and Guoliang X ${ }^{1,2}$
}

\author{
*Correspondence: \\ yuanxianxu@cardc.cn \\ State Key Laboratory of \\ Aerodynamics, China Aerodynamics \\ Research and Development Center, \\ 621000 Mianyang, China \\ ${ }^{2}$ Computational Aerodynamics \\ Institute, China Aerodynamics \\ Research and Development Center \\ 621000 Mianyang, China
}

\begin{abstract}
Properties of wall pressure beneath a transitional hypersonic boundary layer over a $7^{\circ}$ half-angle blunt cone at angle of attack $6^{\circ}$ are studied by Direct Numerical Simulation. The wall pressure has two distinct frequency peaks. The low-frequency peak with $f \approx 10-50 \mathrm{kHz}$ is very likely the unsteady crossflow mode based on its convection direction, i.e. along the axial direction and towards the windward symmetry ray. Highfrequency peaks are roughly proportional to the local boundary layer thickness. Along the trajectories of stationary crossflow vortices, the location of intense high-frequency wall pressure moves from the bottom of trough where the boundary layer is thin to the bottom of shoulder where the boundary layer is thick. By comparing the pressure field with that inside a high-speed transitional swept-wing boundary layer dominated by the z-type secondary crossflow mode, we found that the high-frequency signal originates from the Mack mode and evolves into the secondary crossflow instability.
\end{abstract}

Keywords: Wall pressure, Mack mode, Secondary crossflow instability, Inclined cone

\section{Introduction}

The boundary layers over realistic aircrafts are usually three-dimensional, i.e., there is crossflow perpendicular to the streamwise direction. The instability mechanism is accordingly much more complicated than that in extensively studied two-dimensional boundary layers (e.g. [1-3]), especially in hypersonic conditions. Therefore, in the last decades, considerable efforts have been made towards building a fundamental understanding to the transition of hypersonic three-dimensional boundary layers by wind tunnel experiments [4-9], theoretical analyses [10-13], direct numerical simulations (Chen et al: Transition of hypersonic boundary layer over a yawed blunt cone, submitted) (Chen et al.: Stationary cross-flow breakdown in a high-speed swept-wing boundary layer, submitted) and flight tests (see overview [14]). Nevertheless, much is still unknown.

Due to its simple configuration with practical significance, an inclined (i.e., non-zero angle of attack) straight circular cone has become a canonical model to study the transition of hypersonic boundary layer with crossflow. Stability analyses have found that, at the side of the hypersonic inclined straight circular cone, the Mack mode [15] and the

(C) The Author(s). 2020 Open Access This article is licensed under a Creative Commons Attribution 4.0 International License which permits use, sharing, adaptation, distribution and reproduction in any medium or format, as long as you give appropriate credit to the original author(s) and the source, provide a link to the Creative Commons licence, and indicate if changes were made. The images or other third party material in this article are included in the article's Creative Commons licence, unless indicated otherwise in a credit line to the material. If material is not included in the article's Creative Commons licence and your intended use is not permitted by statutory regulation or exceeds the permitted use, you will need to obtain permission directly from the copyright holder. To view a copy of this licence, visit http://creativecommons.org/licenses/by/4.0/. 
secondary instabilities of crossflow vortices [16] are the two principal high-frequency instability groups $[10,12]$. However, not all the unstable modes identified by the stability analyses will present or appear with equal importance during the natural transition process. A typical example is the breakdown of crossflow vortices. In both the low- and high-speed flows with crossflow, it has been found that the z-type (produced by the spanwise or azimuthal gradients of the streamwise mean flow) secondary crossflow instability is responsible for the natural transition process [17] (Chen et al.: Stationary cross-flow breakdown in a high-speed swept-wing boundary layer, submitted), although stability analyses $[13,17]$ also detected the y-type (produced by the wall-normal gradient of the streamwise mean flow) secondary crossflow instabilities.

In experiments, there is still controversy about the transition routine in the hypersonic boundary layers over inclined straight circular cones. The key point of issue is whether the transition is triggered by the Mack mode or by the z-type secondary crossflow mode. Based on the wall pressure, [4] claimed that the Mack mode was responsible for the transition. In contrast, $[5,7,8]$ preferred that the transition was triggered by the z-type secondary crossflow instability, which is in agreement with [6], who for the first time obtained the mean and fluctuations of velocity fields inside the boundary layer by employing hot-wire probes instead of only measuring wall pressure signals. However, the frequency response in [6] only reaches to $180 \mathrm{kHz}$. It is possible that not all the instabilities had been detected. Meanwhile, however, [8] also speculated that the transition could be attributed to the crossflow modulated Mack mode.

The reason for the above controversy can be largely attributed to the limited information that the surface pressure sensors could afford. Surface pressure sensors have been widely used in wind tunnel experiments and flight tests. The propagation direction, wave length, convection velocity and temporal spectra of instability modes reflected by the wall pressure, for instance, can be measured by these sensors. But usually, wall pressure at very limited positions can be obtained by those sensors instead of the whole pressure field in numerical simulations (e.g. [18-21]). For flow with potential instabilities associated with separate frequency bands, the frequency alone can sometimes be sufficient to detect the principal instability responsible for the transition. However, in the hypersonic three-dimensional boundary layer with crossflow, frequencies of the most amplified Mack mode and the z-type secondary crossflow mode are in the same range [10]. Then it is not trivial to differentiate the Mack mode and the z-type secondary crossflow mode only by frequency $[8,10]$.

The best way to distinguish the secondary crossflow instability modes and the Mack mode is by measuring their eigenfunctions within the boundary layer as [10,12], which is, however, impossible by surface pressure sensors. The Mack mode has a unique characteristic, i.e. its mode shapes of velocity and temperature have two peaks in the wall-normal direction. Li et al. [10] differentiates the two modes by the pressure distribution inside the boundary layer. Specifically, the Mack mode has two pressure extremes, with a stronger one beneath the trough and a weaker one at the shoulder of stationary crossflow vortices. While the z-type secondary crossflow instability only has one pressure extreme at the shoulder of the crossflow vortices. However, recently (Chen et al.: Stationary cross-flow breakdown in a high-speed swept-wing boundary layer, submitted) found that the vortical structure of the z-type secondary crossflow instability in a high-speed swept-wing boundary layer reaches further to the wall, which should be 
reflected by the wall pressure. We will see later that such speculation is proved to be correct.

Understanding the transition mechanism is the prerequisite for control strategy, which depends on the transition routine. For the Mack mode, ultrasonically absorptive coatings or wavy wall are usually used (see overview [22]). For the crossflow modes, plasma actuators [23], localized suction [24] and discrete-roughness-elements [25] are possible options. Consequently, the transition routine in experiments needs to be further confirmed preferably with more information inside the boundary layer. For this purpose, we intend to find what else is required except the wall pressure signals, because the direct numerical simulation is able to offer all the information compared with wind tunnel experiments or flight tests. The present paper studied the wall pressure in a highlyresolved direct numerical simulation of the transition process in a hypersonic boundary layer over a $7^{\circ}$ half-angle straight circular cone at an angle of attack (AoA) $6^{\circ}$. Properties of pressure around the transition front and also along the trajectories of stationary crossflow vortices are compared with those during the natural breakdown of stationary crossflow vortices in a high-speed swept-wing boundary layer in the absence of the Mack mode (Chen et al.: Stationary cross-flow breakdown in a high-speed swept-wing boundary layer, submitted). Hopefully, our results may shed some light on the understanding of measured signals by surface pressure sensors in experiments.

The present work is a companion to [26] (Chen et al.: Transition of hypersonic boundary layer over a yawed blunt cone, submitted), in which the results of stability analyses and the evolution of vortical structures have been presented. To the best knowledge of the authors, the direct numerical simulation in the present paper and [26] (Chen et al.: Transition of hypersonic boundary layer over a yawed blunt cone, submitted) is the largest one for the transition process in hypersonic boundary layer over an inclined straight circular cone.

\section{Numerical simulation}

As shown in Fig. 1, we are considering flow around a straight circular cone at AoA $6^{\circ}$ with half-angle $7^{\circ}$, nose radium $1 \mathrm{~mm}$ and length $700 \mathrm{~mm}$. The free stream conditions are from a wind tunnel experiment with Mach number $M_{\infty}=6$, unit Reynolds number

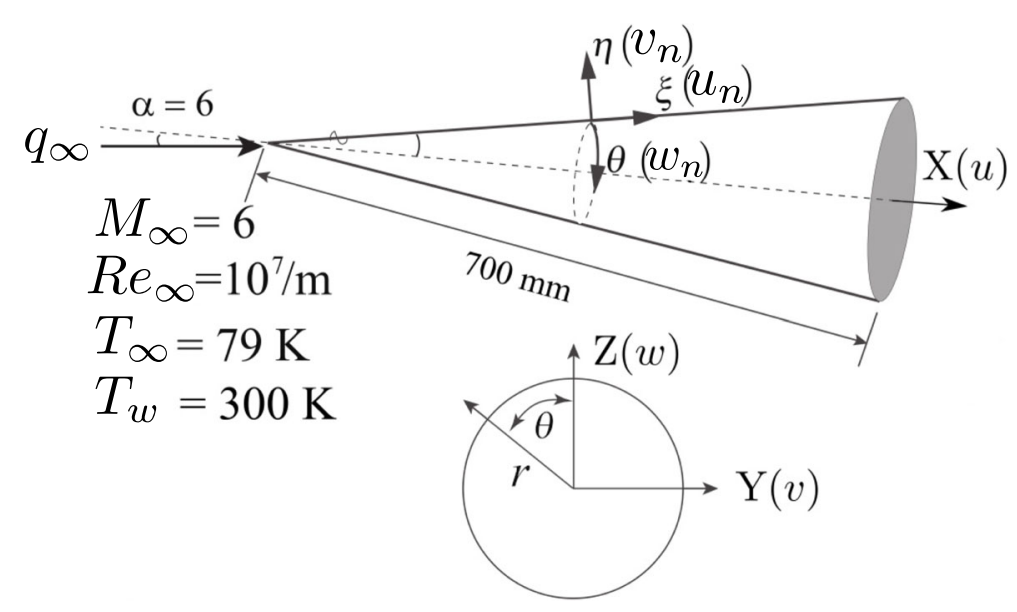

Fig. 1 The sketch of the simulation, notations and coordinates 
$10^{7} / \mathrm{m}$ and temperature $T_{\infty}=79 \mathrm{~K}$. The wall temperature is $300 \mathrm{~K}$. The freestream condition is the same as that in [27], where a blunt cone with similar configuration is placed at $\mathrm{AoA}=1^{\circ}$. The $6^{\circ} \mathrm{AoA}$ is achieved by inclining the freestream velocity vector. Two coordinate systems are introduced, namely the Cartesian coordinate system $(x, y, z)$ with its origin at the circle center of the nose and the local body-fitted orthogonal coordinate system $(\xi, \eta, \zeta)$. Velocity components in the two coordinate systems are respectively $(u, v, w)$ and $\left(u_{n}, v_{n}, w_{n}\right)$. The total velocity is denoted by $q=\sqrt{u^{2}+v^{2}+w^{2}}$. Note that the azimuthal angle $\theta$ changes from $0^{\circ}$ at the leeward symmetry plane to $180^{\circ}$ at the windward symmetry plane, which is the opposite to the definition in most of the experiments.

The simulation procedure in [27] is adopted in the present paper. First, the laminar baseflow including the nose and the shock wave is obtained with a second-order finite volume method with grid size $N_{\xi} \times N_{\eta} \times N_{\zeta}=1000 \times 301 \times 181$. The wall distance of the first layer grid varies linearly from $4 \times 10^{-3} \mathrm{~mm}$ at the nose to $8 \times 10^{-3} \mathrm{~mm}$ at the axial outlet. In this step, only half of the model is used considering the symmetry of the flow. Figure $2 \mathrm{a}$ and $\mathrm{b}$ respectively display the axial slice of nondimensional $u$ and $T$ on the leeward side of the base flow. Due to the pressure gradient, the flow accumulates toward the leeward symmetry plane where mushroom vortex is formed.

Second, a subdomain without the nose $(x \geq 50 \mathrm{~mm})$ and the shock wave is extracted, with the inlet and farfield interpolated from the previous step. At the inlet, the respective distances of the farfield to the wall is about four and two times of the local boundary layer thickness $\delta$ at $\theta=0^{\circ}$ and $180^{\circ}$. Subdomain excluding the nose (leading edge) and the shock is also employed by (Chen et al.: Stationary cross-flow breakdown in a highspeed swept-wing boundary layer, submitted), who studied the break down of stationary crossflow vortices in a high-speed swept-wing boundary layer. In this step, the whole azimuthal domain is computed since the symmetry is not satisfied for instantaneous flow fields. To alleviate the computation cost, a buffer region is used between $\theta=202.5^{\circ}$ and $\theta=337.5^{\circ}$ with coarse grid. A steady and azimuthal random blowing-suction with intensity $0.1 \% q_{\infty}$ is introduced between $90 \mathrm{~mm}<x<100 \mathrm{~mm}$. Ideally, steady blowing-suction can only generate stationary crossflow vortices. But it was only after the simulation finished when we realized that the initial flow field is actually a copy of the inlet (the same as in [27]), which inevitably introduces considerable numerical errors that lead to secondary instabilities of crossflow vortices and the subsequent breakdown process.

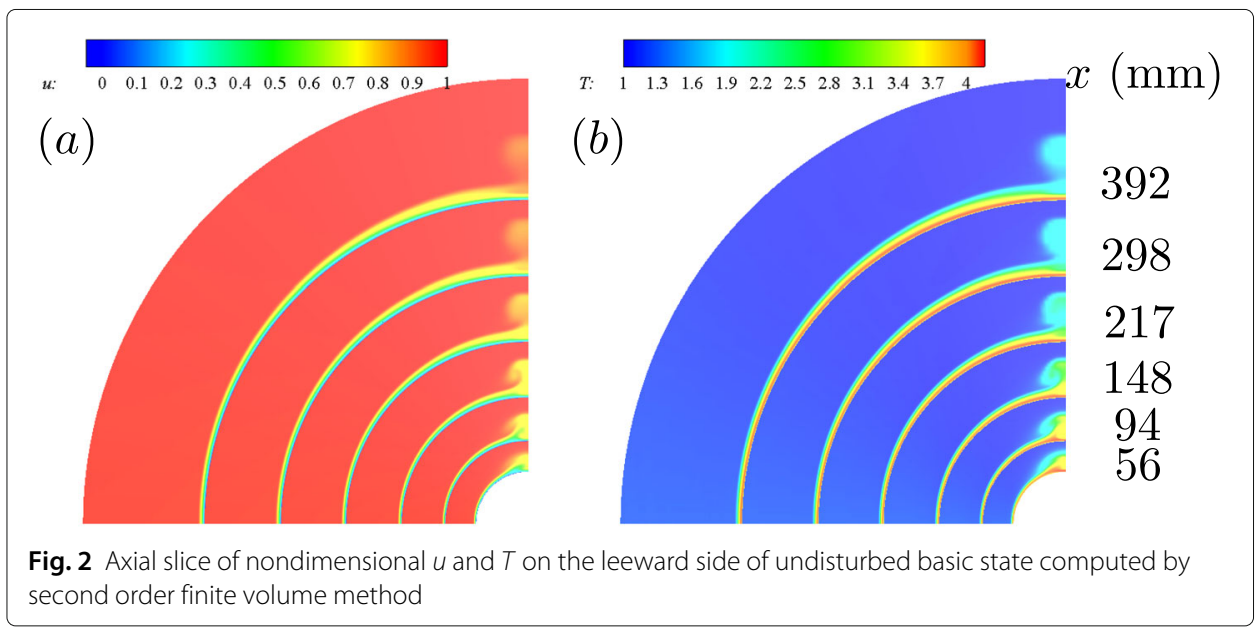


Compressible Navier-Stokes equations in conservative formulation are discretized in the curvature mesh and solved using a widely used parallel software OPENCFD developed by [28]. This code has been validated [27, 28] and used widely in compressible boundary-layer transition and turbulence [29-33]. Non-slip condition is applied at the wall. The inviscous and viscous terms are discretized respectively with modified 7thorder WENO scheme [34] and 6th-order central difference. Time is advanced with explicit third-order Runge-Kutta method. In the wall-normal direction, at each azimuthal ray $\left(k=1,2, \ldots, N_{\zeta}\right)$, exponentially stretched grid points with wall-normal distances satisfying

$$
\eta(i, j)=\frac{\exp \left(\frac{j-1}{N_{\eta}-1} b\right)-1}{\exp (b)-1} L_{\eta}(i, k), i=1,2, \ldots, N_{\xi} \text { and } j=1,2, \ldots, N_{\eta}
$$

are applied to capture strong derivatives near the wall, where $L_{\eta}(i, k)$ is the local wallnormal distance of the farfield to the wall. The factor $b$ is determined by prescribing the wall distance of the first layer grid $\eta(i, 2), i=1,2, \ldots, N_{\xi}\left(10^{-2} \mathrm{~mm}\right.$ in the present work). The grid size excluding the buffer region in the axial and azimuthal directions is $N_{\xi} \times$ $N_{\eta} \times N_{\zeta}=2800 \times 300 \times 1039$, yielding grid resolutions in the axial, wall-normal and azimuthal directions are $\Delta \xi^{+} \approx 10, \Delta \eta_{w}^{+} \approx 0.4$ and $\Delta \zeta^{+} \approx 7-10$ downstream of the transition front, where $\Delta \eta_{w}$ is measured at the wall. Quantities with superscript ' + ' are in the wall units based on the wall-friction velocity $u_{\tau}=\sqrt{\tau_{w} / \rho_{w}}$ and kinematic viscosity $v_{w}=\mu_{w} / \rho_{w}$, where $\tau_{w}=\mu \partial \bar{q}_{n} /\left.\partial \eta\right|_{\eta=0}$ and $\bar{q}_{n}$ is the total mean velocity that is tangent to the wall, where $\bar{\bullet}$ denotes the temporal average.

\section{Results and discussion}

\subsection{Transition front and the temporal spectra of wall pressure}

Since most of the details about the transitional flow fields such as instantaneous snapshots in the wall-parallel or azimuthal planes, vortical structures, temporal spectra inside the boundary layer and stability analyses have been studied thoroughly in [26] (Chen et al.: Transition of hypersonic boundary layer over a yawed blunt cone, submitted). We will only give a brief introduction to the transition process before discussing properties of the wall pressure.

Figure 3 gives an overall picture of the transition process by showing the slices of instantaneous $u$ in wall-normal planes at different axial locations. At the side of the cone, the growth and breakdown of crossflow vortices are clearly observed.

Figure 4 shows the temporal averaged mean wall heat flux

$$
\bar{Q}_{w}=\left.2 \frac{\mu_{w} C_{p}}{\operatorname{RePr}} \partial_{\eta} T\right|_{\eta=0}
$$

on the unrolled cone surface, where $C_{p}=\gamma / M_{\infty}^{2}(\gamma-1)$ with $\gamma$ the specific heat ratio and the Prandtl number $\mathrm{Pr}=0.7$. In Fig. 4, $\zeta$ represents the distance with respect to the $\theta=90^{\circ}$ ray in terms of the arc length. The windward and leeward symmetry planes are respectively at the lower and upper limits of $\zeta$. The transition onset locates at $x \approx 300$ $\mathrm{mm}$ and $\theta \approx 52^{\circ}$, yielding $R e_{x}=3 \times 10^{6}$, which is close to that in a Mach 8 noisy wind tunnel experiment [7]. Obviously, $\bar{Q}_{w}$ appears in streaky patterns on the leeward side $\left(\theta<90^{\circ}\right)$, suggesting that the transition is due to the breakdown of stationary crossflow vortices in that area. The dashed lines represent trajectories of two representative stationary crossflow vortices determined by the local maximum of $\bar{Q}_{w}$. The trajectories are 


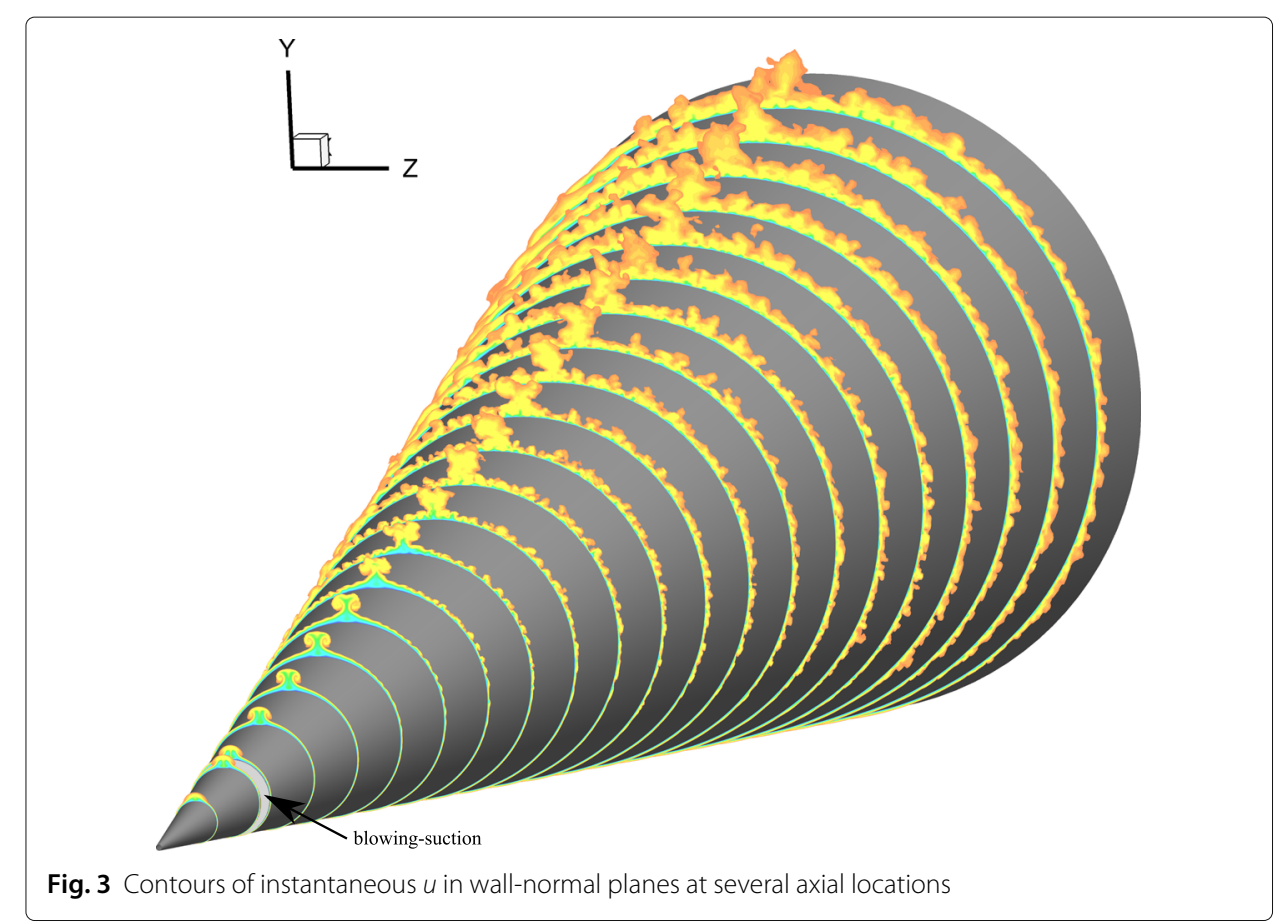

roughly inclined by $12-13^{\circ}$ with respect to the $\theta=90^{\circ}$ ray, which is consistent with both experiments [35] and numerical simulations $[10,36]$ under similar conditions. The axial evolution of $\bar{Q}_{w}$ along the two trajectories are given in Fig. 4b, suggesting that the two stationary crossflow vortices grow exponentially between $x=260 \mathrm{~mm}$ and $320 \mathrm{~mm}$. On the windward side $\left(\theta>90^{\circ}\right)$, the transition is highly possible caused by unsteady modes.

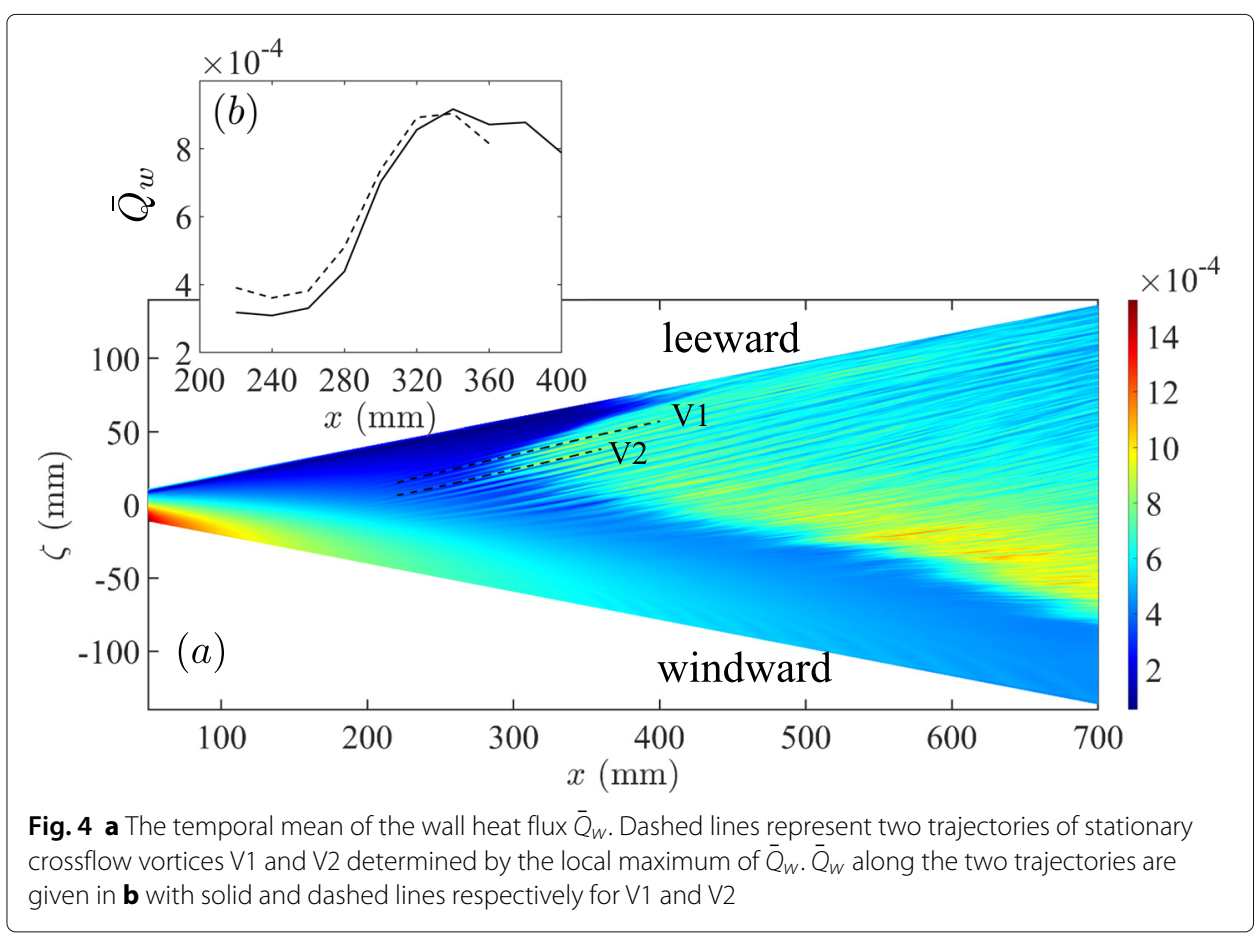




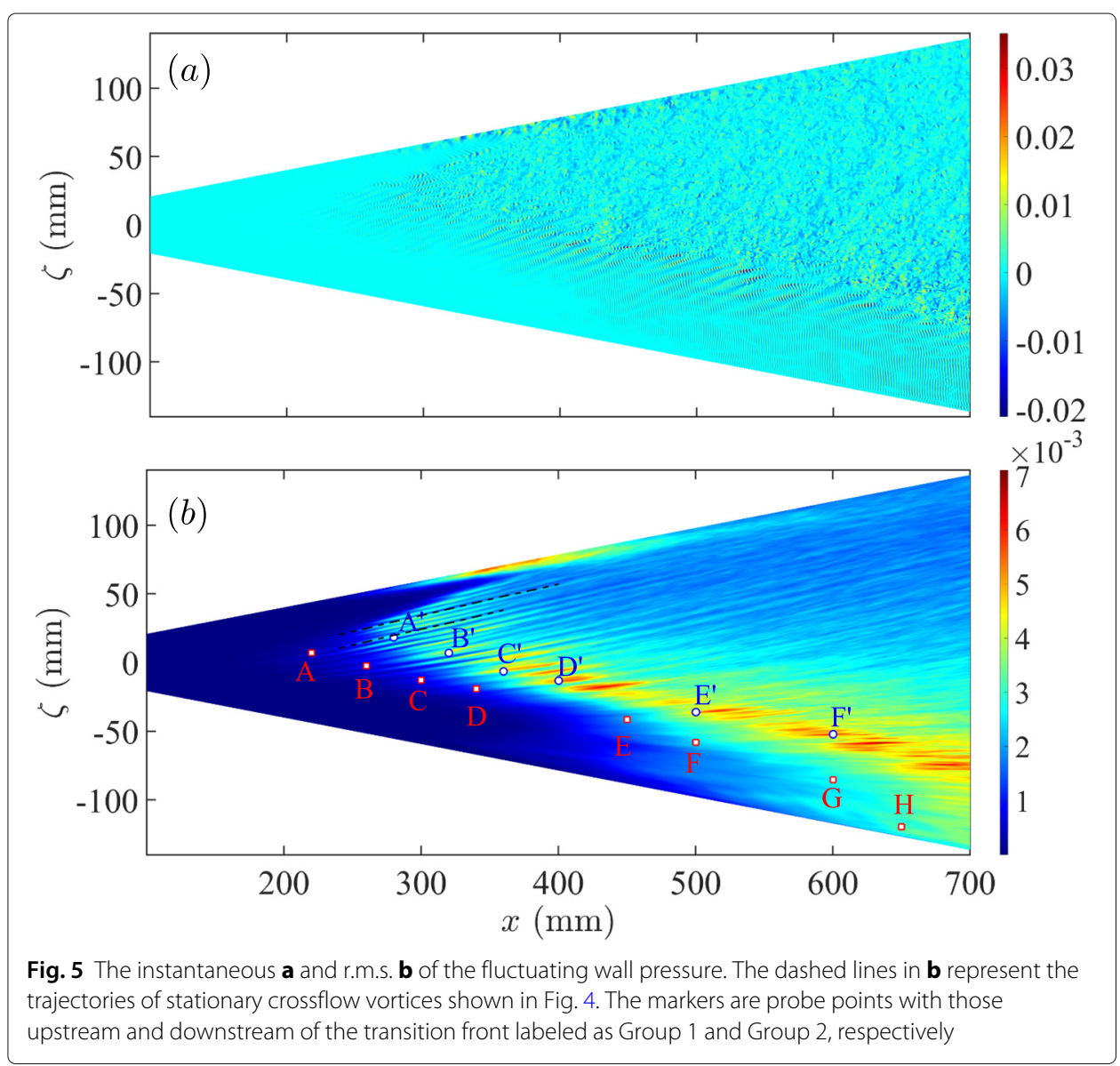

We will focus on the wall pressure

$$
p_{w}=\frac{\rho_{w} T_{w}}{\gamma M_{\infty}^{2}}
$$

hereinafter. $p_{w}$ is sampled with a frequency $\approx 3.2 \mathrm{MHz}$, which should be sufficient to capture all the possible instabilities at the side. The duration of the sampling is roughly two times of the go-through period $x_{L} / q_{\infty}$. Results do not change when using half of the samples.

Figure 5a shows one instantaneous snapshot of fluctuating $p_{w}$. Upstream of the transition front (where the wall heat flux grows violently in Fig. 4a), especially beyond $x=400$ $\mathrm{mm}$, there are quasi two-dimensional structures, indicating the origin of the Mack mode, whose presence is justified by quasi two-dimensional vortical structures shown in (Chen et al: Transition of hypersonic boundary layer over a yawed blunt cone, submitted). Wall

Table 1 The coordinates of probe points in the Group 1 (upstream of the transition front) and Group 2 (downstream of the transition front)

\begin{tabular}{lllllllll}
\hline Group 1 & $\mathrm{A}$ & $\mathrm{B}$ & $\mathrm{C}$ & $\mathrm{D}$ & $\mathrm{E}$ & $\mathrm{F}$ & $\mathrm{G}$ & $\mathrm{H}$ \\
$x(\mathrm{~mm})$ & 220 & 260 & 300 & 340 & 450 & 500 & 600 & 650 \\
$\theta\left(^{\circ}\right)$ & 75.7 & 94.3 & 109.4 & 115.8 & 132.3 & 143.3 & 155.5 & 174.7 \\
Group 2 & $\mathrm{A}^{\prime}$ & $\mathrm{B}^{\prime}$ & $\mathrm{C}^{\prime}$ & $\mathrm{D}^{\prime}$ & $\mathrm{E}^{\prime}$ & $\mathrm{F}^{\prime}$ & & \\
$x(\mathrm{~mm})$ & 280 & 320 & 360 & 400 & 500 & 600 & \\
$\theta\left({ }^{\circ}\right)$ & 60 & 80 & 98 & 105 & 123 & 130 & \\
\hline
\end{tabular}




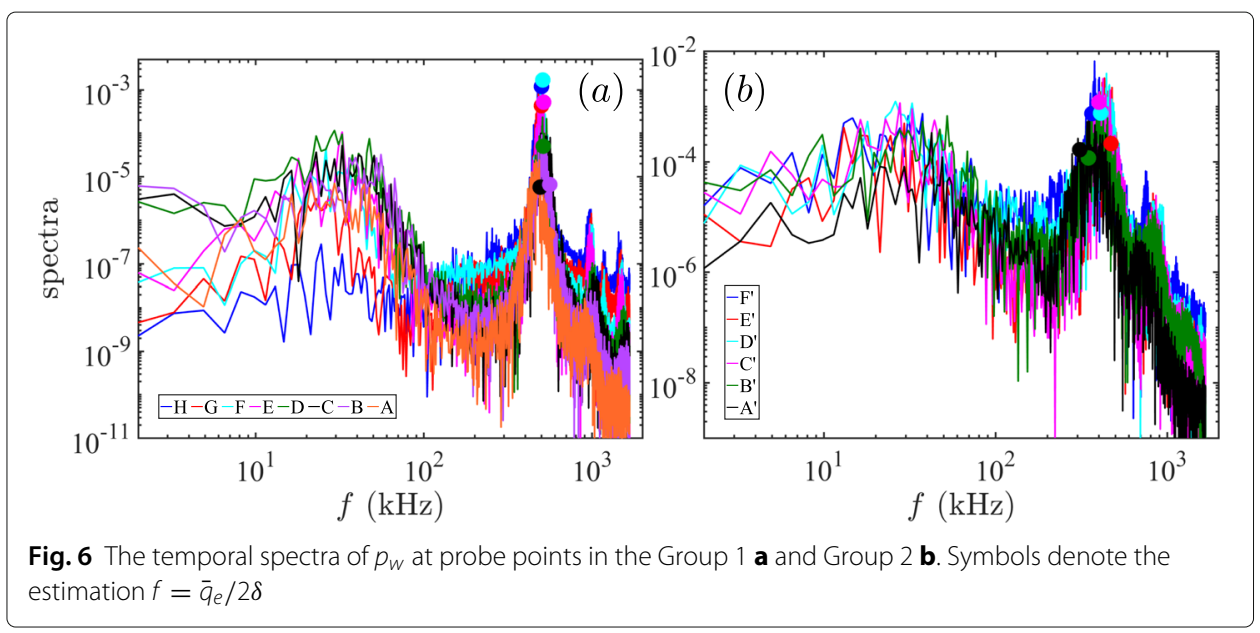

pressure of similar pattern was also identified by [27] on the surface of a cone placed at $\mathrm{AoA}=1^{\circ}$ with the same freestream condition. Figure $5 \mathrm{~b}$ displays the r.m.s. of the wall pressure. Similar to the wall heat flux in Fig. $4 \mathrm{a},\left(p_{w}\right)_{\text {rms }}$ is also trapped by stationary crossflow vortices at $\theta \lesssim 90^{\circ}$. We will investigate the properties of $p_{w}$ at several probe points marked in Fig. 5b around the transition front. They are divided into two groups, namely the Group 1 and Group 2 respectively upstream and downstream of the transition front. Their coordinates are tabulated in the Table 1.

Figure 6 shows the temporal spectra of $p_{w}$ at these probe points. Two obvious peaks can be observed, i.e., a low-frequency one centered around $30 \mathrm{kHz}$ independent of the locations. And a high-frequency one centered at $450-500 \mathrm{kHz}$ for probe points in Group 1 and $350-450 \mathrm{kHz}$ in Group 2. The frequencies at high-frequency peaks roughly follow the estimation $f=\bar{q}_{e} / 2 \delta$, which are given by the filled circles in the figure. The highfrequency peaks downstream of the transition front are broader, which is likely due to nonlinear effects. Harmonics of the high-frequency peaks are also clearly seen, indicating the presence of self interactions.

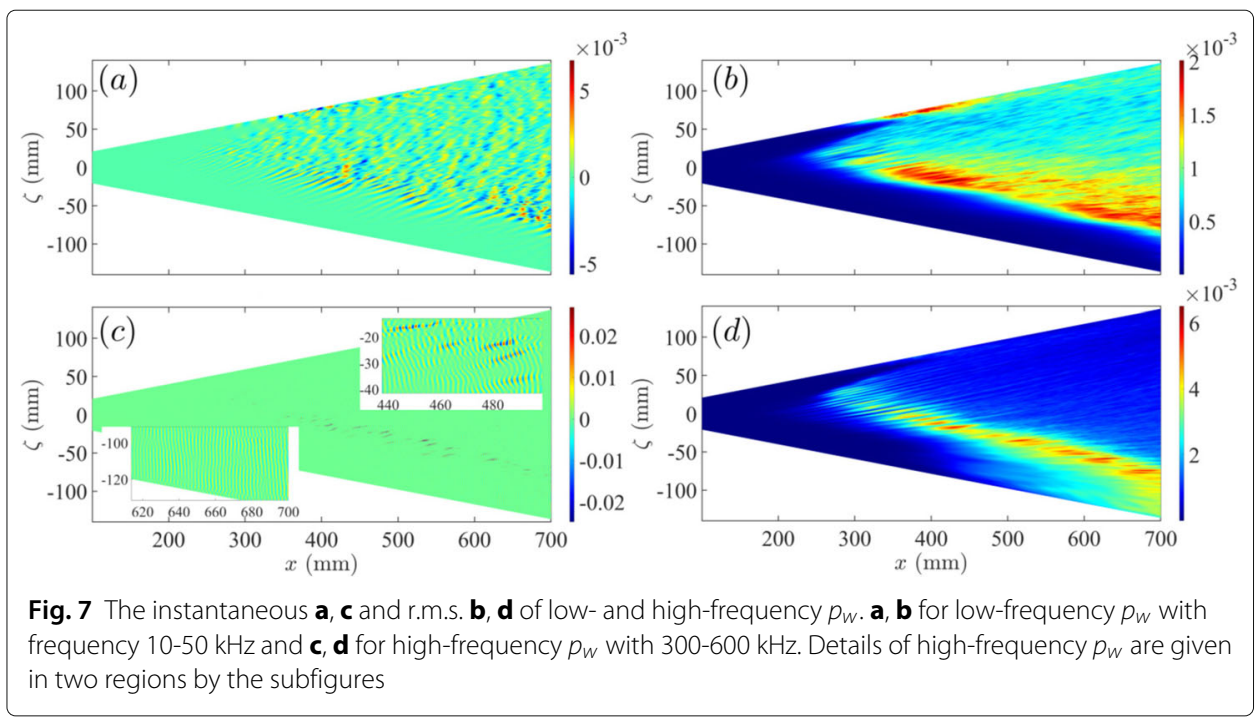


Based on the above temporal spectra, we further filtered $p_{w}$ by a cut-off filter with frequency bands $10-50 \mathrm{kHz}$ and $300-600 \mathrm{kHz}$. The instantaneous $p_{w}$ of low and high frequencies are shown in Fig. 7a, c. The low-frequency $p_{w}$ exhibits large-scale streaky structures, roughly following the transition front in Fig. 4a, while the high-frequency $p_{w}$ shows small-scale wavepacket structures. Moreover, the wavepackets reside closer to the windward ray than the streaks, especially at the rear of the cone, where they are almost two-dimensional. The combined low- and high-frequency $p_{w}$ form a pattern that small-scale wavepackets ride on large-scale streaks in the vicinity of the transition front as shown in Fig. 5a. The resulting $\left(p_{w}\right)_{\text {rms }}$ of low- and high-frequency $p_{w}$ are given in Fig. $7 b, d$.

\subsection{Distribution of fluctuating pressure inside stationary crossflow vortices}

For a sharp, $7^{\circ}$ half-angle cone with $6^{\circ}$ AoA in a Mach 6 quiet wind tunnel, [8] found that the high-frequency $(175-325 \mathrm{kHz})$ wall pressure disturbances coincide with the local maximum of the wall heat flux. While the low-frequency wall pressure $(100-175 \mathrm{kHz})$ disturbances lie between heat streaks. They argued that if the high-frequency signal is the crossflow modified Mack mode, then the local maximum of $\left(p_{w}\right)_{\text {rms }}$ should be under the thin trough of crossflow vortex where the wall heat flux is high, as they had observed. [4] also detected high-frequency signals, which were regarded as the Mack mode, in the position where the boundary layer is thin.

In a similar way to [8], Fig. 8 displays $\left(p_{w}\right)_{\mathrm{rms}}$ and $\bar{Q}_{w}$ in the neighbourhood of the trajectories of stationary crossflow vortices V1 and V2. Dashed lines in the figure correspond to the local maximum of $\bar{Q}_{w}$. There is no particular pattern between $\left(p_{w}\right)_{\text {rms }}$ of low-frequency $p_{w}$ and $\bar{Q}_{w}$ (Fig. 8a-b, I). Specifically, around V1, $\left(p_{w}\right)_{\text {rms }}$ has no preference about the heat flux, while around $\mathrm{V} 2,\left(p_{w}\right)_{\mathrm{rms}}$ lies between heat streaks upstream of $x \approx 260 \mathrm{~mm}$, while it coincides with the heat peaks when stationary crossflow vortices are strong. But for high-frequency $p_{w}$, Fig. 8a-b, II clearly shows that the spatially localized $\left(p_{w}\right)_{\text {rms }}$ presented in Fig. 7 is concentrated under the peak heating areas up to $x \approx 280$ $\mathrm{mm}$, beyond where $\left(p_{w}\right)_{\mathrm{rms}}$ and $\bar{Q}_{w}$ deviate.

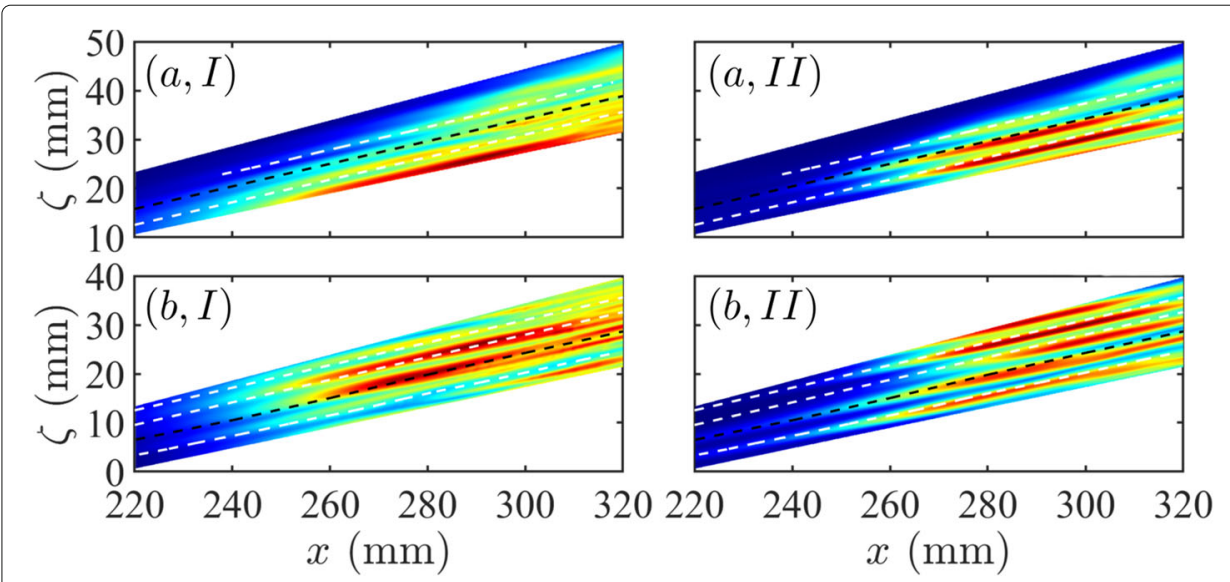

Fig. 8 The distribution of the r.m.s. (shaded contour) of the low- (10-50 kHz, I) and high-frequency (300-600 $\mathrm{kHz}, \mathrm{II}$ ) wall pressure and the wall heat flux (dashed lines) in the neighbourhood of stationary crossflow vortex trajectories $\mathrm{V} 1$ a and $\mathrm{V} 2 \mathbf{b}$. Trajectories $\mathrm{V} 1$ and $\mathrm{V} 2$ are colored in black 

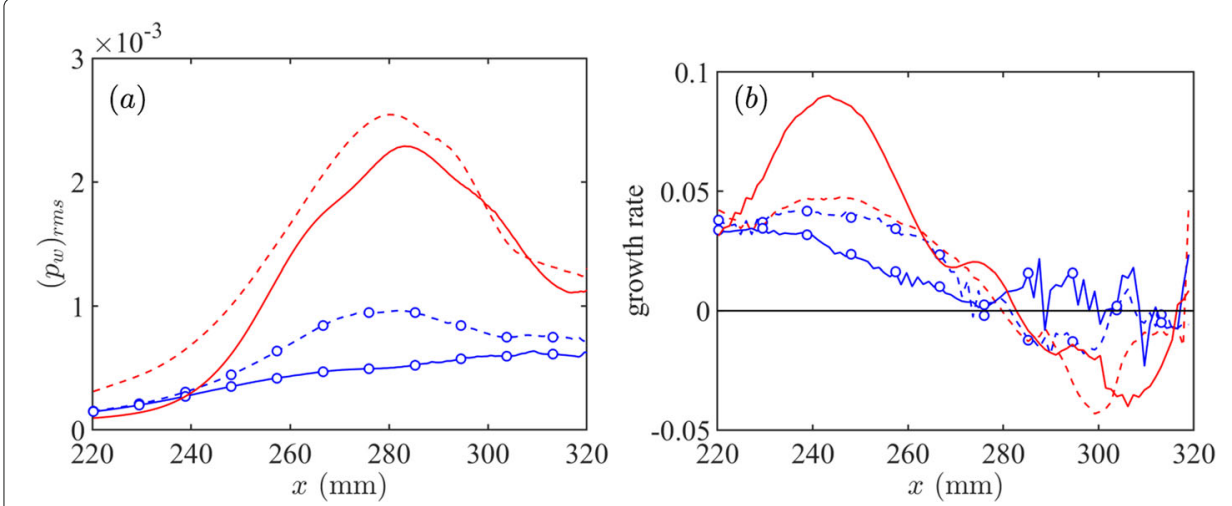

Fig. 9 Axial evolution of $\left(p_{w}\right)_{\text {rms }}(\mathbf{a})$ and its growth rate $(\mathbf{b})$ along the stationary crossflow vortex trajectories V1 (solid) and V2 (dashed). Lines with and without symbols are respectively for the low- and high-frequency wall pressure

Figure 9a shows the amplitude evolution of $\left(p_{w}\right)_{\mathrm{rms}}$ along the trajectories V1 and V2. The corresponding growth rates

$$
\mathrm{d} \ln \left[\left(p_{w}\right)_{\mathrm{rms}}\right] / \mathrm{d} x_{\text {vortex }}
$$

are shown in Fig. 9b. High-frequency $p_{w}$ along V1 experience the most violent growth, while others only grow mildly, especially for the low-frequency $p_{w}$ along V1. This dependence of growth rates on the trajectories of stationary crossflow vortices is probably due to the inhomogeneity in the azimuthal direction. The peaks of $\left(p_{w}\right)_{\text {rms }}$ arrive at $x \approx 280$ $\mathrm{mm}$, where the wall heat flux starts to grow exponentially as indicated by Fig. $4 \mathrm{~b}$.

Since we have all the information inside the boundary layer, Fig. 10 further shows the evolution of $p_{\text {rms }}$ inside the boundary layer along the vortex trajectory $\mathrm{V} 2$ at several axial locations from $x=220 \mathrm{~mm}$ to $320 \mathrm{~mm}$. $p_{\text {rms }}$ has its maximum near the wall, distributed locally in the azimuthal direction even when the boundary layer has not been markedly modulated by the stationary crossflow vortex yet (Fig. 10a). But when the rolling up of stationary crossflow vortex becomes prominent, it turns out that intense $p_{\text {rms }}$ locates roughly beneath the trough of crossflow vortex, where the boundary layer is thin. However, when the stationary crossflow vortex is about to saturate (Fig. 10e-f) as indicated by Fig. $4 \mathrm{~b}$,

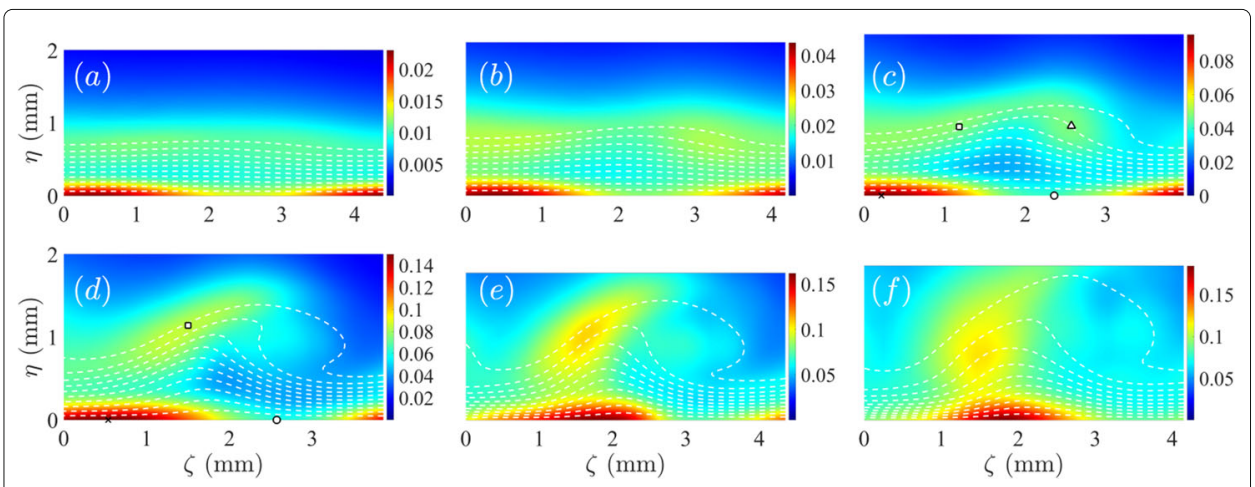

Fig. 10 The evolution of $p_{\text {rms }}$ in the wall-normal plane along the vortex path $V 2$. The dashed lines represent the temporal mean axial velocity. The markers in $\mathbf{c}, \mathbf{d}$ are probe points. Axial locations are from $x=220 \mathrm{~mm}$ to $320 \mathrm{~mm}$ by an increment of $20 \mathrm{~mm}$ 

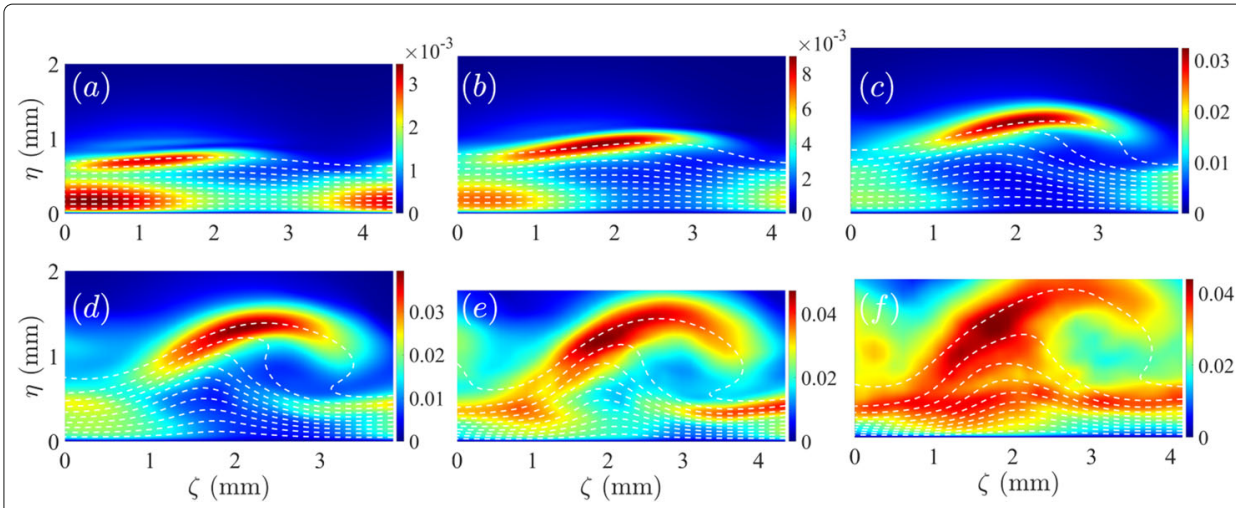

Fig. 11 The same as Fig. 10 but for the r.m.s. of axial velocity with frequency between $250 \mathrm{kHz}$ and $550 \mathrm{kHz}$

the maximum of near-wall $p_{\text {rms }}$ shifts to the bottom of the shoulder, where the boundary layer is thicker and the wall heat transfer is accordingly weaker. Meanwhile, another weaker peak of $p_{\text {rms }}$ emerges at the shoulder. The evolution of $p_{\text {rms }}$ inside the boundary layer gives a more clear explanation about the relation between $\left(p_{w}\right)_{\text {rms }}$ and $\bar{Q}_{w}$.

The distribution of $p_{\text {rms }}$ inside the boundary layer when stationary crossflow vortices are still weak agrees well with that of the extracted Mack mode by stability analysis [10]. It means that the high-frequency signals here are in fact the Mack mode, which is supported by the distribution of the r.m.s. of the axial velocity shown in Fig. 11. The r.m.s. of the axial velocity inside the boundary layer obviously has two peaks, which is the unique feature of the Mack mode.

Also in a Mach 6 quiet wind tunnel, [5] found that when pressure sensors at the side of an inclined cone are rotated by a small azimuthal angle $\left(\lesssim \pm 5^{\circ}\right)$. The measured peak frequency with $f \approx 400 \mathrm{kHz}$ disappears, whereas a lower frequency peak with $f \approx 150 \mathrm{kHz}$ appears. Edelman and Schneider [8] also found similar phenomenon. They speculated that the frequency jump probably can be ascribed to the relative position between sensors and crossflow vortices. While in a Mach 8 conventional wind tunnel [7], such frequency jump was not observed. Instead, the peak frequency only decreases smoothly as a function of the local boundary layer thickness. The frequency jump observed by $[5,8]$ is also examined in the present work. Results are shown in Fig. 12, which displays the temporal
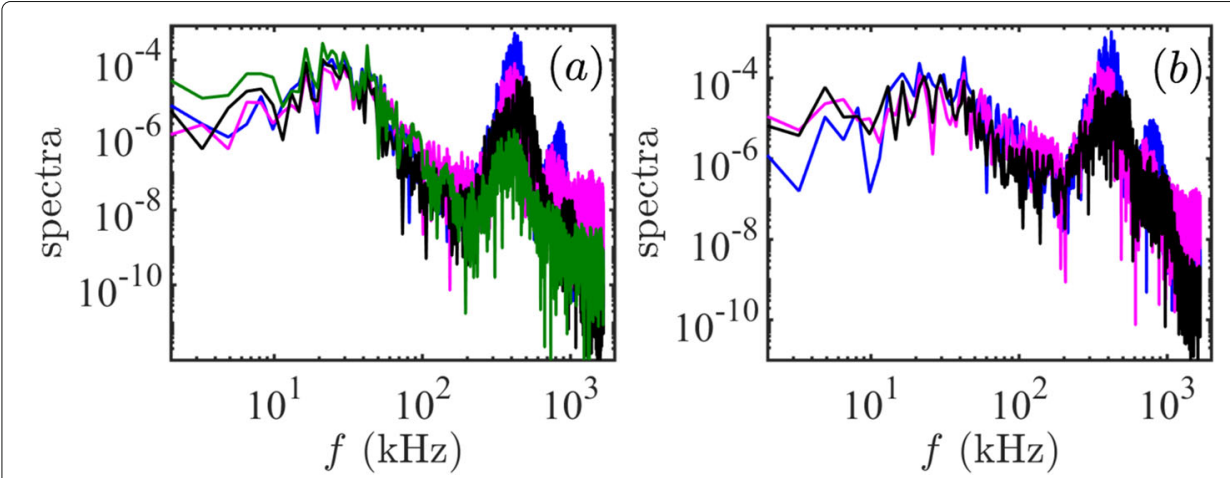

Fig. 12 The temporal spectra measured at the probe points marked in Fig. 10c, d. $\mathbf{a} x=260 \mathrm{~mm}$ and $\mathbf{b}$ $x=280 \mathrm{~mm}$. Lines are __ for $x, \ldots$ for $\bigcirc, \ldots$ for $\square$ and __ for $\triangle$ 
spectra at different locations inside a stationary crossflow vortex following the trajectory V2 shown in Fig. 10c, d. The two probes at the wall have a difference of azimuthal angle by $\approx 3.5^{\circ}$. Apparently, inside this crossflow vortex, the peak frequency does not vary with the azimuthal angle, neither with the wall-normal distance. But the intensities at the highfrequency peaks vary significantly, which is also indicated in Fig. 7d. The same conclusion holds along the vortex path V1. Our results are consistent with [7], probably because the present transition process also happens in a noisy environment.

Based on the results shown in Figs. 8 and 10, probably what $[4,8]$ observed between $\left(p_{w}\right)_{\text {rms }}$ and $\bar{Q}_{w}$ were in the region where crossflow vortices were still weak. The relative position between pressure footprint and stationary crossflow vortices during the whole transition process shown above needs to be further verified by experiments.

\subsection{Space-time correlation}

In this section, we study the space-time correlation coefficient of wall pressure defined by

$$
C_{p p}\left(\Delta_{\xi}, \Delta_{\zeta}, \Delta_{t}\right)=\frac{\overline{p_{w}\left(\xi^{\prime}, \zeta^{\prime}, t^{\prime}\right) p_{w}(\xi, \zeta, t)}}{\left(p_{w}\right)_{\mathrm{rms}}\left(\xi^{\prime}, \zeta^{\prime}, t^{\prime}\right)\left(p_{w}\right)_{\mathrm{rms}}(\xi, \zeta, t)},
$$

where $\Delta_{\xi}=\xi-\xi^{\prime}$ and $\Delta_{\zeta}=\zeta-\zeta^{\prime}$ are respectively the spatial separations in the axial and the azimuthal directions and $\Delta_{t}=t-t^{\prime}$ is the time delay. $\left(\xi^{\prime}, \zeta^{\prime}\right)$ is the position of the reference point marked in Fig. $5 \mathrm{~b}$ and $(\xi, \zeta)$ is the position of moving points. $\Delta_{\zeta}<0$ means the moving point is closer to the windward symmetry plane than the reference point, while the opposite is true for $\Delta_{\zeta}>0 . C_{p p}\left(\Delta_{\xi}, \Delta_{\zeta}, 0\right)$ reveals the spatial organization of $p_{w}$, while $C_{p p}\left(\Delta_{\xi}, 0, \Delta_{t}\right)$ or $C_{p p}\left(0, \Delta_{\zeta}, \Delta_{t}\right)$ tells the temporal propagation in the space. The space-time correlation has been widely used in turbulent boundary layers (e.g. $[18,19])$.

We computed $C_{p p}\left(\Delta_{\xi}, \Delta_{\zeta}, \Delta_{t}\right)$ for low- and high-frequency $p_{w}$ separately. The spatial correlation coefficients $C_{p p}\left(\Delta_{\xi}, \Delta_{\zeta}, 0\right)$ are presented first. The results for low-frequency $p_{w}$ at probe points in Group 1 are shown in Fig. 13. The inclination angle $\alpha_{p}$ of the lowfrequency $p_{w}$ streaks or the angle of the wave front against the $\theta=90^{\circ}$ meridian is roughly $22^{\circ}$ except near the rear of the cone, where $\alpha_{p} \approx 26^{\circ}$. This angle is very close to that of low-frequency wall pressure disturbances detected in a Mach 6 wind tunnel

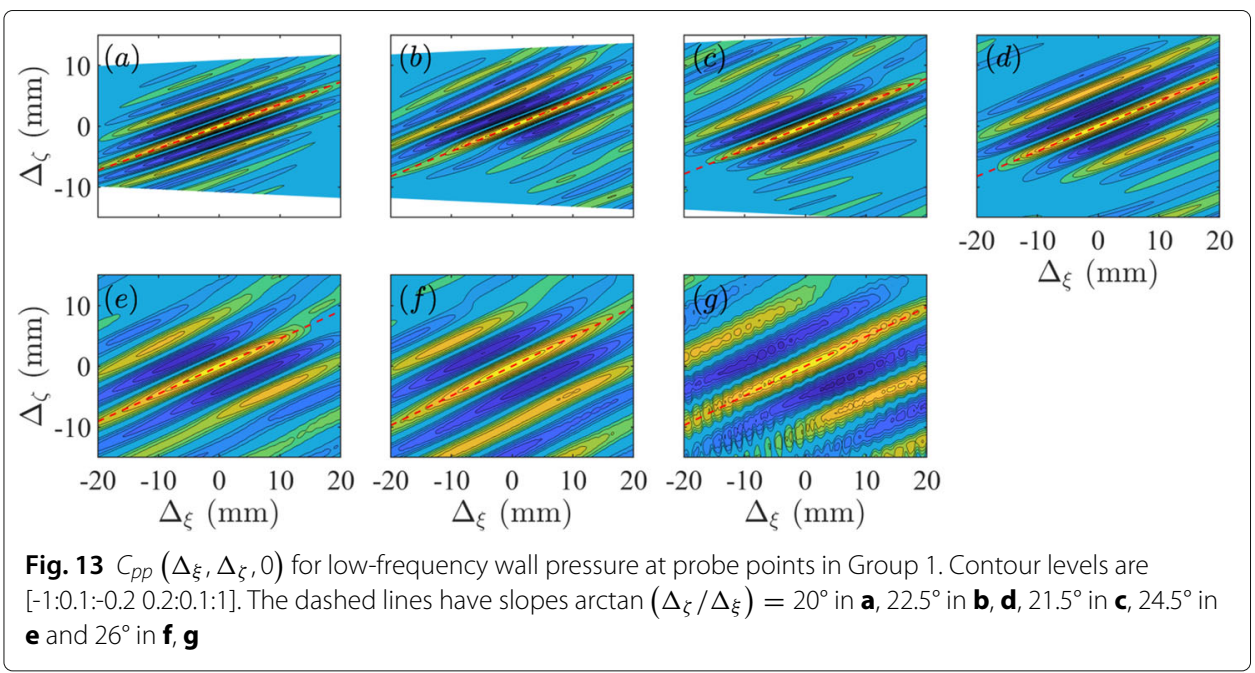




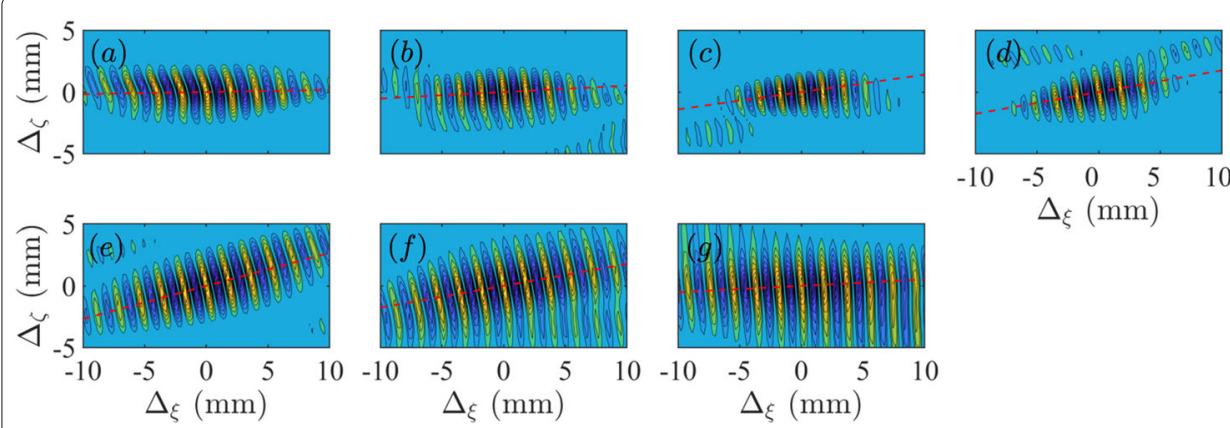

Fig. $14 C_{p p}\left(\Delta_{\xi}, \Delta_{\zeta}, 0\right)$ for high-frequency wall pressure at probe points in Group 1. Contour levels are the same as in Fig. 13. The dashed lines have slopes $\arctan \left(\Delta_{\zeta} / \Delta_{\xi}\right)=1^{\circ}$ in $\mathbf{a}, 3^{\circ}$ in $\mathbf{b}, 8^{\circ}$ in $\mathbf{c}, 10^{\circ}$ in $\mathbf{d}, 15^{\circ}$ in $\mathbf{e}$, $15^{\circ}$ in $\mathbf{f}$ and $3^{\circ}$ in $\mathbf{g}$

[4]. The length $l_{t}$ of the low-frequency $p_{w}$ streak in its inclined direction is roughly 27$40 \mathrm{~mm}$, taking $C_{p p}\left(\Delta_{\xi}, \Delta_{\zeta}, 0\right)=0.5$. The distance $l_{n}$ between low-frequency $p_{w}$ streaks increases from $\approx 2.1 \mathrm{~mm}$ to $\approx 4.8 \mathrm{~mm}$ as a function of the axial positions, measured by the distance between the positive and negative lobes normal to the inclined direction. The low-frequency $p_{w}$ at probe points in Group 2 have similar inclination angles (not shown) as those in Group 1, except for higher noise-to-signal ratio since more small scales are expected to generate downstream of the transition front. The length $l_{t}$ of low-frequency $p_{w}$ streak is in the range $\approx 21-32 \mathrm{~mm}$ and the distance between low-frequency $p_{w}$ streaks $l_{n}$ increases from $\approx 2.7 \mathrm{~mm}$ to $\approx 4.2 \mathrm{~mm}$ as a function of the axial positions.

On the other hand, the spatial organization of high-frequency $p_{w}$ displayed in Figs. 14 and 15 , is totally different from that of low-frequency $p_{w}$, as we have already noticed in the instantaneous snapshots shown in Fig. 7. High-frequency $p_{w}$ appears as wavepackets, with the wavelength measured by the distance between the consecutive positive lobes roughly double of the local boundary layer thickness, as tabulated in the Table 2, which is the feature of the Mack mode [37]. At those positions closer to the windward symmetry plane (Fig. 14f-g), the wavepackets tend to be nearly two-dimensional, with the azimuthal length roughly five times of the wavelength. The size of the wavepackets shrinks significantly in both the axial and azimuthal directions downstream of the transition front. For instance, the axial length of the wavepacket is roughly $20 \mathrm{~mm}$ at probe points in Group 1 , and it decreases to $10 \mathrm{~mm}$ in Group 2. The inclination angles $\alpha_{p}$ of the high-frequency

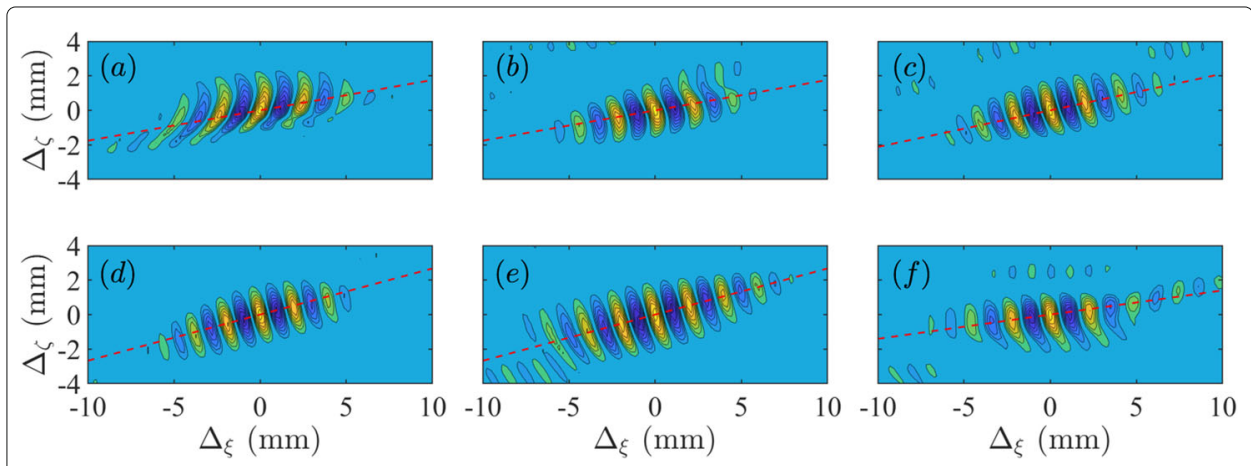

Fig. $15 C_{p p}\left(\Delta_{\xi}, \Delta_{\zeta}, 0\right)$ for high-frequency wall pressure at probe points in Group 2. Contour levels are the same as in Fig. 13. The dashed lines have slopes $\arctan \left(\Delta_{\zeta} / \Delta_{\xi}\right)=10^{\circ}$ in $\mathbf{a}, \mathbf{b}, 12^{\circ}$ in $\mathbf{c}, 15^{\circ}$ in $\mathbf{d}$, e and $8^{\circ}$ in $\mathbf{f}$ 
Table 2 The boundary layer thickness $\delta$ and the wavelength $\lambda$ of high-frequency $p_{w}$ at probe points in Group 1 (upstream of the transition front) and in Group 2 (downstream of the transition front)

\begin{tabular}{llllllll}
\hline Group 1 & A & B & C & D & E & F & G \\
$\delta(\mathrm{mm})$ & 1.03 & 1.03 & 1.01 & 1.00 & 1.02 & 1.06 & 0.98 \\
$\lambda(\mathrm{mm})$ & 2.0 & 1.85 & 1.75 & 1.8 & 1.77 & 1.74 & 1.86 \\
Group 2 & $\mathrm{A}^{\prime}$ & $\mathrm{B}^{\prime}$ & $\mathrm{C}^{\prime}$ & $\mathrm{D}^{\prime}$ & $\mathrm{E}^{\prime}$ & $\mathrm{F}^{\prime}$ & \\
$\delta(\mathrm{mm})$ & 1.424 & 1.1 & 1.27 & 1.30 & 1.52 & 1.71 & \\
$\lambda(\mathrm{mm})$ & 2.43 & 2.23 & 2.13 & 2.05 & 2.07 & 2.24 & \\
\hline
\end{tabular}

wavepackets with respect to the $\theta=90^{\circ}$ ray are represented by the dashed lines. $\alpha_{p}$ are listed in the Table 3 and compared to those of flow at the edge of boundary layer

$$
\alpha_{e}=\operatorname{atan}\left(\bar{w}_{n} /\left.\bar{u}_{n}\right|_{\eta=\delta}\right),
$$

and to those of near-wall flow at the first layer of the grid

$$
\alpha_{w}=\operatorname{atan}\left(\bar{w}_{n} /\left.\bar{u}_{n}\right|_{\eta=\eta_{w}}\right) .
$$

$\alpha_{p}$ does not seem to follow either $\alpha_{w}$ or $\alpha_{e}$ but is roughly in the middle of them. $\alpha_{w}$ is larger than $\alpha_{e}$, indicating that the streamlines near the wall are more inclined to the leeward ray than those at the boundary edge.

Next, the temporal propagation is presented. The results for low-frequency $p_{w}$ at probe points in Group 2 are shown in Figs. 16 and 17 respectively for $C_{p p}\left(0, \Delta_{\zeta}, \Delta_{t}\right)$ and $C_{p p}\left(\Delta_{\xi}, 0, \Delta_{t}\right)$. The shape of the contours reflects the convective nature of pressure signals. The convection velocity $U_{c}$ can be estimated by the ratio $\Delta_{\xi}\left(\Delta_{\zeta}\right) / \Delta_{t}$ at a given time delay $\Delta_{t}$ with the maximum of $C_{p p}$ obtained at $\Delta_{\xi}\left(\Delta_{\zeta}\right)$. When $U_{c}$ is not a function of $\Delta_{t}$ as in the present case, the convection velocity can be estimated by the slopes of the contour lines, as indicated by the dashed lines. The low-frequency $p_{w}$ is convected from the leeward side to the windward side, with a convection velocities ranging from $0.175 q_{\infty}$ to $0.25 q_{\infty}$. In the axial direction, the convection velocity is about $0.45-0.50 q_{\infty}$, yielding a propagation angle roughly between $-29^{\circ}$ and $-19^{\circ}$. Similar values can be found for those probe points in the Group 1 thus results are not shown.

In summary, the low-frequency $p_{w}$ travels downstream and toward the windward symmetry plane, which is in the expected direction for travelling crossflow modes. Muñoz et al. [4] believed that the low-frequency signal measured in experiments travelling in the same manner maybe either the first-mode instability mode or the travelling crossflow mode. Whereas [7] preferred that they are actually the noise in the wind tunnel. However, in quiet wind tunnels, $[5,6,8]$ believed that they are the travelling crossflow modes.

Table 3 The inclination angles of the high-frequency $p_{w}\left(\alpha_{p}\right)$ and those of the wall parallel velocity components at the edge of the boundary layer $\left(\alpha_{e}\right)$ and the first layer of grid $\left(\alpha_{w}\right)$

\begin{tabular}{llllllll}
\hline Group 1 & $\mathrm{A}$ & $\mathrm{B}$ & $\mathrm{C}$ & $\mathrm{D}$ & $\mathrm{E}$ & $\mathrm{F}$ & $\mathrm{G}$ \\
$\alpha_{e}\left(^{\circ}\right)$ & 1.01 & 2.32 & 3.39 & 4.29 & 5.49 & 5.87 & 6.62 \\
$\alpha_{W}\left(^{\circ}\right)$ & 4.33 & 9.17 & 13.56 & 17.29 & 22.48 & 24.37 & 28.07 \\
$\alpha_{p}\left(^{\circ}\right)$ & 1.0 & 3.0 & 8.0 & 10.0 & 15.0 & 15.0 & 3.0 \\
Group 2 & $\mathrm{A}^{\prime}$ & $\mathrm{B}^{\prime}$ & $\mathrm{C}^{\prime}$ & $\mathrm{D}^{\prime}$ & $\mathrm{E}^{\prime}$ & $\mathrm{F}^{\prime}$ & 7.06 \\
$\alpha_{e}\left(^{\circ}\right)$ & 4.46 & 4.98 & 6.17 & 6.43 & 6.94 & 2.30 \\
$\alpha_{W}\left({ }^{\circ}\right)$ & 15.80 & 19.45 & 22.95 & 23.40 & 24.30 & 11.87 \\
$\alpha_{p}\left(^{\circ}\right)$ & 10.0 & 10.0 & 12.0 & 15.0 & 15.0 & 8 \\
\hline
\end{tabular}




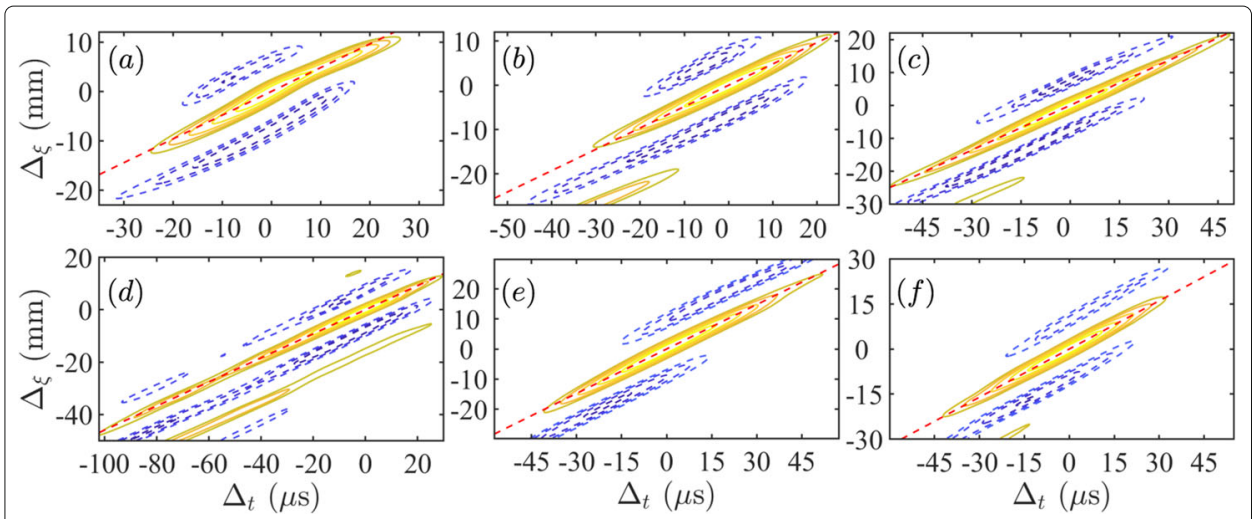

Fig. $16 C_{p p}\left(\Delta_{\xi}, 0, \Delta_{t}\right)$ for low-frequency wall pressure at probe points in Group 2. Contour levels are [0.5:0.1:1] for solid and [-1:0.1:-0.5] for dashed. The slopes of the dashed lines are $0.45 q_{\infty}$ in $\mathbf{a}, 0.5 q_{\infty}$ in $\mathbf{b}$, $0.425 q_{\infty}$ in $\mathbf{c}, 0.43 q_{\infty}$ in $\mathbf{d}, 0.46 q_{\infty}$ in e and $0.45 q_{\infty}$ in $\mathbf{f}$

High-frequency $p_{w}$ are convected in the axial direction much faster than the lowfrequency ones, with a convection velocity $U_{c} \approx 0.85 q_{\infty}\left(\approx 0.85 \bar{q}_{e}\right)$, independent of the position as shown by $C_{p p}\left(\Delta_{\xi}, 0, \Delta_{t}\right)$ in Fig. 18 for those probe points in the Group 2. While in the azimuthal direction, $C_{p p}\left(0, \Delta_{\zeta}, \Delta_{t}\right)$ is almost vertical, yielding a convection velocity up to $10 q_{\infty}$ at the probe point $\mathrm{F}^{\prime}$ and around $4 q_{\infty}$ at other points (not shown). Such a high velocity is probably only mathematical and non-physical. Because with such a velocity, the time for travelling one millimeter is less than $0.25 / q_{\infty}$, which is already smaller than our sampling time interval $0.32 / q_{\infty}$. We prefer that the results only reflect the azimuthal coherence of $p_{w}$ when convected as a wavepackets along the axial direction, instead of the realistic propagation in the azimuthal direction.

The above results (Figs. 16, 17 and 18) only show $C_{p p}\left(\Delta_{\xi}, 0, \Delta_{t}\right)$ and $C_{p p}\left(0, \Delta_{\zeta}, \Delta_{t}\right)$ for probe points in the Group 2, i.e., downstream of the transition front. The convection velocities for both low- and high-frequency $p_{w}$ in the Group 1 are respectively similar to those in the Group 2. But the average window for computing the correlation coefficient, i.e., the extent of $\Delta_{\xi}$ and $\Delta_{t}$ is much larger, especially for large-scale low-frequency $p_{w}$ near the rear of the cone.

\subsection{The footprints of genuine z-type secondary crossflow instability}

We have seen in Figs. 8 and 10 that intense $p_{\text {rms }}$ shifts from the bottom of trough to the bottom of shoulder with the development of stationary crossflow vortices. We believe that

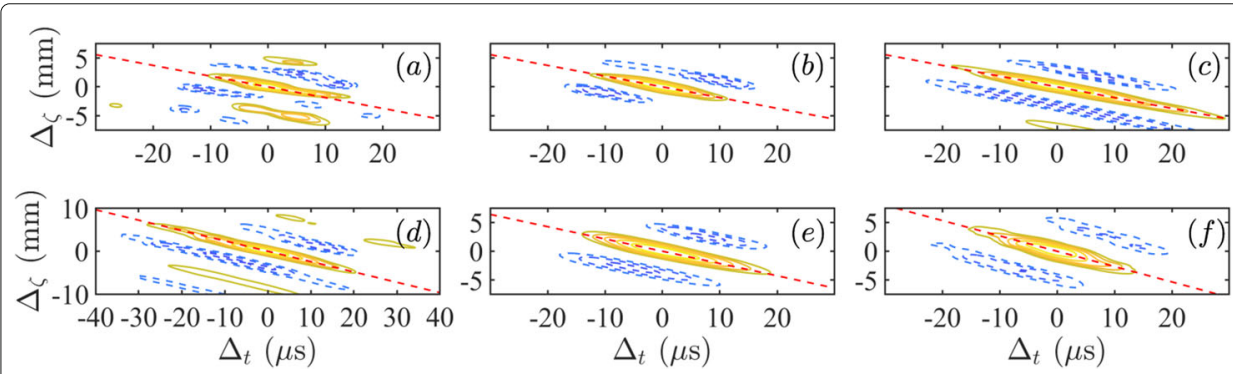

Fig. $17 C_{p p}\left(0, \Delta_{\zeta}, \Delta_{t}\right)$ for low-frequency wall pressure at probe points in Group 2. Contour levels are the same as in Fig. 16 . The slopes of the dashed lines are $-0.175 q_{\infty}$ in $\mathbf{a}-\mathbf{c}_{,}-0.225 q_{\infty}$ in $\mathbf{d},-0.2 q_{\infty}$ in $\mathbf{e}$ and $-0.25 q_{\infty}$ in $\mathbf{f}$ 

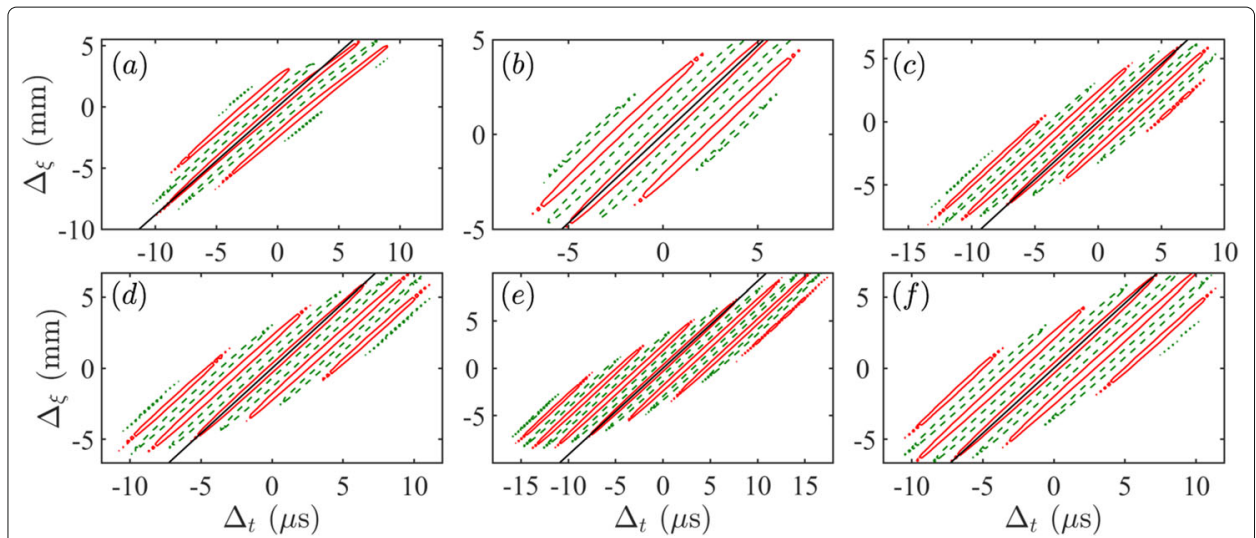

Fig. $18 C_{p p}\left(\Delta_{\xi}, 0, \Delta_{t}\right)$ for high-frequency wall pressure at probe points in Group 2. Solid and dashed contours for 0.5 and -0.5 respectively. Solid lines represent $\Delta_{\xi} / \Delta_{t}=0.85 q_{\infty}$

the high-frequency signals originate from the Mack mode and evolve into the secondary crossflow mode as they are modulated by the crossflow vortices. In other words, it may suggest that the pressure footprint of genuine z-type secondary crossflow instability is beneath the shoulder of crossflow vortices.

Recently, (Chen et al.: Stationary cross-flow breakdown in a high-speed swept-wing boundary layer, submitted) investigated the natural transition process in a high-speed swept-wing boundary layer. The transition is triggered by the z-type secondary crossflow instability riding on stationary crossflow vortices. In that work, the freestream Mach number is 6, but the Mach number at the boundary edge is less than four. No unstable Mack modes were identified by stability analysis. Therefore, the properties of pressure in (Chen et al.: Stationary cross-flow breakdown in a high-speed swept-wing boundary layer, submitted) probably could be representative for the genuine z-type secondary crossflow instability in the hypersonic condition. Readers are referred to (Chen et al.: Stationary cross-flow breakdown in a high-speed swept-wing boundary layer, submitted) for more details about the setup of numerical simulation and how the z-type secondary crossflow instability is excited.

First, we show the distribution of $p_{\text {rms }}$ in wall-normal planes at three axial locations with $x=200 \mathrm{~mm}, 230 \mathrm{~mm}$ and $260 \mathrm{~mm}$ in Fig. 19. $x=230 \mathrm{~mm}$ and $260 \mathrm{~mm}$ roughly correspond to the saturation point of stationary crossflow vortices and the disturbances excited by the blowing-suction, respectively (Chen et al.: Stationary cross-flow breakdown in

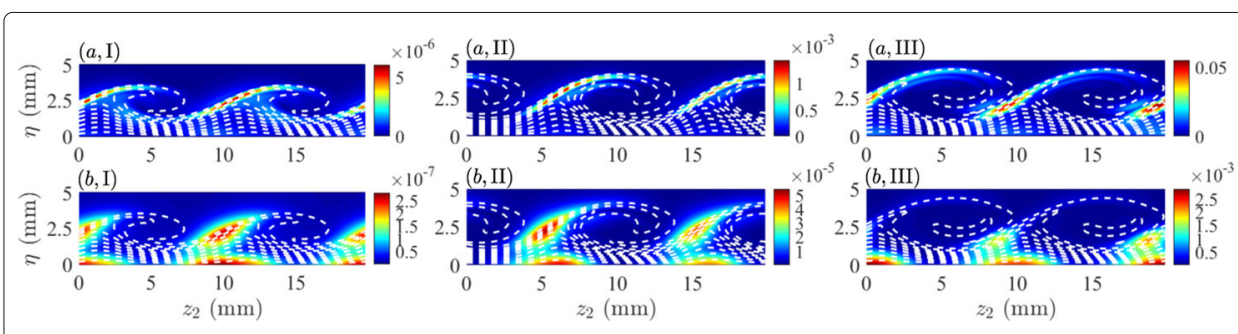

Fig. 19 The evolution of $u_{2, \mathrm{rms}}(\mathbf{a}, I-I I I)$ and $p_{\mathrm{rms}}(\mathbf{b}, \mathrm{I}, \mathrm{III})$ in the wall-normal planes at $x=200 \mathrm{~mm}(\mathrm{I}), 230 \mathrm{~mm}$ (II) and $260 \mathrm{~mm}$ (III) during the breakdown of stationary crossflow vortices in a high-speed swept wing boundary layer (Chen et al.: Stationary cross-flow breakdown in a high-speed swept-wing boundary layer, submitted). The dashed lines represent the temporal mean of the vortex-axial oriented velocity $u_{2}$ 

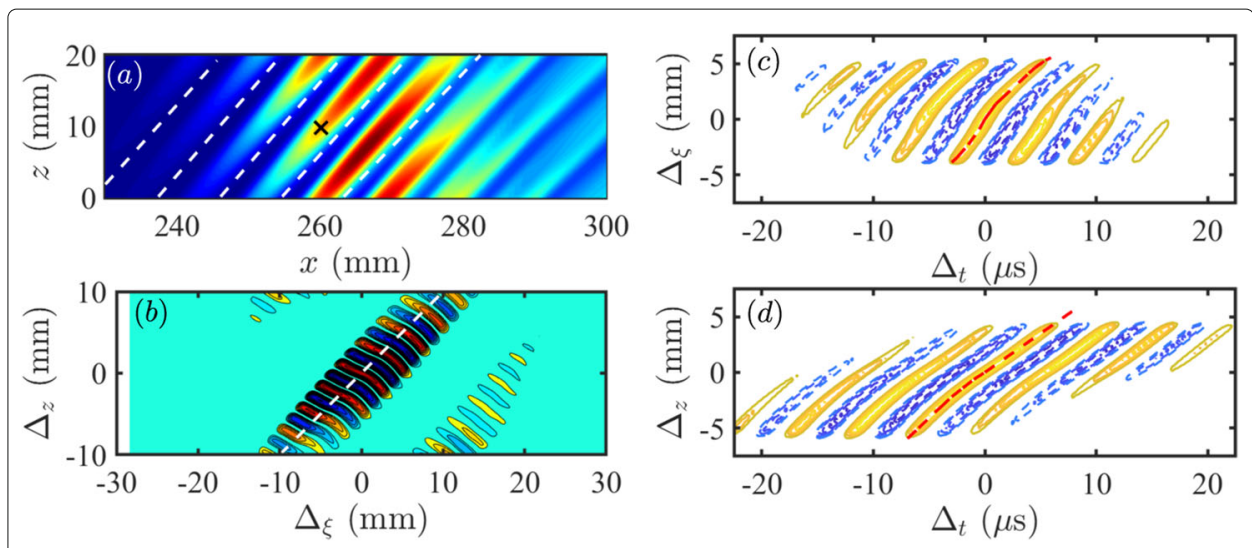

Fig. 20 a The r.m.s. of the wall-pressure. Dashed lines represent the local maximum of the mean skin-friction coefficient; $\mathbf{b}$ The spatial correlation coefficient $C_{p p}\left(\Delta_{\xi}, \Delta_{z}, 0\right)$ at the probe point in $\mathbf{a}$. Contours lines are [-1:0.1:-0.2 0.2:0.1:1]. The dashed line has a slope by $45^{\circ} ; \mathbf{c}$ and $\mathbf{d}$ are respectively $C_{p p}\left(\Delta_{\xi}, 0, \Delta_{t}\right)$ and $C_{p p}\left(\Delta_{z}, 0, \Delta_{t}\right)$. Contours levels are [-1:0.1:-0.5 0.5:0.1:1]. Dashed lines are the best fit for the center of the contour

a high-speed swept-wing boundary layer, submitted). It is clear that in the high-speed swept-wing boundary layer, the pressure is distributed separately in two regions: one at the shoulder of crossflow vortex coinciding with the r.m.s. of the vortex-axial oriented velocity and another one just below the shoulder and close to the wall with similar intensity. Although they are spatially separated, the peak frequencies at the two $\left(p_{w}\right)_{\text {rms }}$ lobes are the same to that of the vortex-axial oriented velocity, indicating that they are of the same thing, or more precisely, they are different parts of the z-type secondary crossflow instability. Obviously, the pressure extreme of the z-type secondary crossflow instability beneath the shoulder contradicts with [10]. The reason for the difference is unclear.

Next, Fig. 20a shows the r.m.s. of the pressure beneath the high-speed swept-wing boundary layer. Since there is only one principal frequency, we did not filter the pressure field. Note that the wall is adiabatic in (Chen et al.: Stationary cross-flow breakdown in a high-speed swept-wing boundary layer, submitted), thus the dashed lines in Fig. 20a represent the local maximum of the skin-friction coefficient $C_{f}$ instead of the wall heat flux. With the presence of stationary crossflow vortices, the streaky $\left(p_{w}\right)_{\text {rms }}$ in Figs. 20a and 8 are not intrinsically different. But $\left(p_{w}\right)_{\text {rms }}$ and $C_{f}$ are always separated because the former lies beneath the thicker part of boundary layer as shown in Fig. 19.

The spatial correlation coefficient of $p_{w}$ at the probe point $(x=260 \mathrm{~mm})$ in Fig. 20a is shown in Fig. 20b. The wave length of $p_{w}$ is about $\delta$, where $\delta$ is defined by the wall-normal height where $\bar{w}(x)$ reaches $0.99 w_{\infty}$. This is different from high-frequency $p_{w}$ we have seen in Figs. 14 and 15 , whose wavelength $\lambda \approx 2 \delta$. The spanwise direction of the swept-wing boundary layer is periodic, thus both of the axial and spanwise extent of the wavepacket reaches to $40 \mathrm{~mm}$, which is roughly 10 times of the local boundary layer thickness. The wavepacket as a whole is inclined with respect to the axial direction by $45^{\circ}$, which is equal to the swept angle. But the high-correlation lobes do not follow the dashed line.

The temporal propagation of the $p_{w}$ beneath the high-speed swept wing boundary layer is shown in Fig. 20c, d. As indicated by the dashed lines, the convection velocity $U_{c}$ is not constant. The general trend is that $p_{w}$ propagates slower with time, in both streamwise and spanwise directions. Specifically, $U_{c, \xi}$ roughly varies between $1.0 \bar{q}_{e}$ to $2.0 \bar{q}_{e}$ and $U_{c, z}$ 
varies roughly between $0.8 \bar{q}_{e}$ to $0.96 \bar{q}_{e}$. We are not clear why the high-frequency signals on the cone surface only propagate in the axial direction.

What we observed in Figs. 19 and 20a is different from the evolution of $p_{\mathrm{rms}}$ along the trajectories of stationary crossflow vortices shown in Figs. 8 and 10e, f. It further verifies that the high-frequency disturbances on the side of the cone have its origin from the Mack mode, which is then modulated by crossflow vortices and turns into the secondary crossflow instability. This probably can be inferred from [10], who found that the modulated Mack mode was more highly amplified than the unmodulated Mack mode. And the Mack mode grows much upstream and achieves higher $N$-factors than the z-type crossflow secondary instability.

\section{Conclusions}

We have conducted a direct numerical simulation of the transition process of a Mach 6 boundary layer over a blunt cone with half-angle $7^{\circ}$ placed at AoA $6^{\circ}$. The transition is achieved by disturbing the boundary layer with a blowing-suction at the wall. The pressure field around the transition front is investigated and compared with that in a transitional high-speed swept-wing boundary layer without unstable Mack mode. The main conclusion is that the transition is due to the crossflow modulated Mack mode, instead of the genuine secondary crossflow modes. This probably also holds for experiments in quiet tunnels or flight tests.

The wall pressure has two apparent frequency peaks. The low-frequency peak has a frequency $f \approx 10-50 \mathrm{kHz}$, independent of the axial and azimuthal locations. Disturbances of the low-frequency peaks manifest as large-scale streaks inclined by $\approx 20^{\circ}-26^{\circ}$ against the $\theta=90^{\circ}$ ray. They propagate downstream and toward the windward symmetry plane, with respective convection velocities $0.45-0.50 q_{\infty}$ and $0.175-0.25 q_{\infty}$. They are likely the travelling crossflow mode.

The high-frequency peak roughly follows the relationship of $f \approx \bar{q}_{e} / 2 \delta$. The highfrequency wall pressure is small-scale and quasi two-dimensional wavepackets with wavelength $\lambda \approx 2 \delta$, indicating that they are the Mack mode. They are convected in the axial direction by a velocity $0.85 q_{\infty}$. When stationary crossflow vortices are still weak, intense $\left(p_{w}\right)_{\text {rms }}$ lies beneath the trough of the stationary crossflow vortices where the wall heat flux (isothermal wall) or the skin friction coefficient is high. With the development of stationary crossflow vortices, the Mack mode is modulated. Meanwhile, the intense $\left(p_{w}\right)_{\text {rms }}$ shifts to the bottom of the shoulder where the boundary layer is thicker, which is the same to that of the z-type secondary crossflow instability in a high-speed swept-wing boundary layer without the Mack mode.

Finally, to determine the origin of the high-frequency signals measured by the surface pressure sensors, [8] and our results suggest that comparing the locations of the maximum wall heat flux and the maximum $\left(p_{w}\right)_{\text {rms }}$ could be the simplest way based only on the wall information in experiments. 


\section{Funding}

This work was supported by the National Key Research and Development Program of China 2016 YFA0401200 and 2019YFA0405200, the National Numerical Wind tunnel (NNW) project, and National Natural Science Foundation of China under contract 11702307.

\section{Availability of data and materials}

All the data for the current study are available from the corresponding author on reasonable request.

\section{Competing interests}

The authors declare that they have no competing interests.

Received: 17 August 2020 Accepted: 16 November 2020

Published online: 14 December 2020

\section{References}

1. Su C, Zhou H (2007) Stability analysis and transition prediction of hypersonic boundary layer over a blunt cone with small nose bluntness at zero angle of attack. Appl Math Mech 28:563-572

2. Hader C, Fasel HF (2019) Three-dimensional wave packet in a Mach 6 boundary layer on a flared cone. J Fluid Mech 885:3

3. Paredes P, Choudhari MM, Li F (2020) Mechanism for frustum transition over blunt cones at hypersonic speeds. J Fluid Mech 894:22

4. Muñoz F, Heitmann D, Radespiel R (2014) Instability modes in boundary layers of an inclined cone at Mach 6. J Spacecr Rocket 51:442-454

5. Ward CAC, Henderson RO, Schneider SP (2015) Possible secondary instability of stationary crossflow vortices on an inclined cone at Mach 6. AIAA Paper 46:2773-2913

6. Craig SA, S. SW (2016) Crossflow instability in a hypersonic boundary layer. J Fluid Mech 808:224-244

7. Edelman JB, Casper KM, Henfling JF, Spillers RW (2017) Crossflow transition on a pitched cone at Mach 8. AIAA Paper 46:2899-2913

8. Edelman JB, Schneider SP (2018) Secondary instabilities of hypersonic stationary crossflow waves. AIAA J 56:182-192

9. Neel IT, Leidy AN, Tichenor NR, Bowersox RDW (2018) Influence of environmental disturbances on hypersonic crossflow instability on the HIFiRE-5 elliptic cone. In: SciTech Forum, AIAA. p 1821. https://doi.org/10.2514/6.20181821

10. Li F, Choundhari M, Paredes P, Duan L (2016) High-frequency instabilities of stationary crossflow vortices in a hypersonic boundary layer. Phys Rev Fluids 1:053603

11. Paredes P, Gosse R, Theofilis V, Kimmel R (2016) Linear modal instabilities of hypersonic flow over an elliptic cone. J Fluid Mech 804:442-466

12. Moyes AJ, Paredes P, Kocian TS, Reed HL (2017) Secondary instabilitiy analysis of crossflow on a hypersonic yawed straight circular cone. J Fluid Mech 812:370-397

13. Xu G, Chen J, Dong S, Fu S (2019) The secondary instabilities of stationary cross-flow vortices in a Mach 6 swept wing flow. J Fluid Mech 873:914-941

14. Kimmel RL, W. AD, Borg MP, S. JJ, Juliano TJ, A. SS, T. BK (2019) First and fifth hypersonic international flight research experiment's flight and ground tests. J Spacecr Rocket 56(2):421-431

15. Mack LM (1984) Boundary-layer linear stability theory. AGARD Rep. 709

16. Malik MR, Li F, Choudhari MM, Chang C (1999) Secondary instability of crossflow vortices and swept-wing boundary layer transition. J Fluids Mech 399:85-115

17. Bonfigli G, Kloker M (2007) Secondary instability of crossflow vortices: validation of the stability theory by direct numerical simulation. J Fluid Mech 583:229-272

18. Bernardini M, Pirozzoli S (2011) Wall pressure fluctuations beneath supersonic turbulent boundary layers. Phys Fluids 23:085102

19. Bernardini M, Pirozzoli S, Grasso F (2011) The wall pressure signature of transonic shock/boundary layer interaction. J Fluid Mech 671:288-312

20. Choi H, Moin P (1990) On the space-time characteristics of wall-pressure fluctuations. Phys Fluids 2(8):1450

21. Ritos K, Drikakis D, Kokkinakis IW (2019) Acoustic loading beneath hypersonic transitional and turbulent boundary layers. J Sound Vib 441:50-62

22. Fedorov AV (2015) Prediction and control of laminar-turbulent transition in high-speed boundary-layer flows. Procedia IUTAM 14:3-14

23. Dörr PC, Kloker MJ (2016) Transition control in a three-dimensional boundary layer by direct attenuation of nonlinear crossflow vortices using plasma actuators. Intl J Heat Fluid Flow 61:449-465

24. Friederich T, Kloker MJ (2012) Control of the secondary cross-flow instability using localized suction. J Fluid Mech 706:470-495

25. Malik M, Liao W, Li F, Choudhari M (2015) Discrete-roughness-element-enhanced swept-wing natural laminar flow at high Reynolds numbers. AIAA J 53:2321-2334

26. Chen X, Chen J, Dong S, Xu G, X. Y (2020) Stability analyses of leeward streamwise vortices for a hypersonic yawed cone at 6 degree angle of attack. Acta Aerodynamica Sin 38(2):299-307

27. Li X, Fu D, Ma Y (2010) Direct numerical simulation of hypersonic boundary layer transition over a blunt cone with a small angle of attack. Phys Fluids 22:025105

28. Li X, Fu D, Ma Y (2008) Direct numerical simulation of hypersonic boundary-layer transition over a blunt cone. AIAA J 46:2899-2913

29. Zhang Y, Bi W, Hussain F, She Z (2014) A generalized Reynolds analogy for compressible wall-bounded turbulent flows. J Fluid Mech 739:392-420 
30. Zheng W, Yang Y, Chen S (2016) Evolutionary geometry of Lagrangian structures in a transitional boundary layer. Phys Fluids 28:035110

31. Tong FL, Li XL, Duan YH, Yu CP (2017) Direct numerical simulation of supersonic turbulent boundary layer subjected to a curved compression ramp. Phys Fluids 29:125101

32. Chen X, Huang GL, Lee CB (2019) Hypersonic boundary layer transition on a concave wall: stationary Görtler vortices. $J$ Fluid Mech 865:1-40

33. Zheng W, Ruan S, Yang Y, He L, Chen S (2019) Image-based modeling of the skin-friction coefficient in compressible boundary-layer transition. J Fluid Mech 875:1175-1203

34. Jiang G, Shu C (1996) Efficient implementation of weighted ENO schemes. J Comput Phys 126:202

35. Swanson EO, Schneider SP (2010) Boundary layer transition on cones at angle of attack in a Mach-6 quiet tunnel. Paper, AIAA:1062

36. Gronvall JE, Johnson HB, Candler GV (2012) Hypersonic three-dimensional boundary transition on a cone at angle of attack. Paper, AIAA:2822

37. Mack LM (1987) Stability of axisymmeric boundary layers on sharp cones at hypersonic Mach numbers. AIAA Conference Paper 87:1413

\section{Publisher's Note}

Springer Nature remains neutral with regard to jurisdictional claims in published maps and institutional affiliations.

- fast, convenient online submission

- thorough peer review by experienced researchers in your field

- rapid publication on acceptance

- support for research data, including large and complex data types

- gold Open Access which fosters wider collaboration and increased citations

- maximum visibility for your research: over $100 \mathrm{M}$ website views per year

At $\mathrm{BMC}$, research is always in progress.

Learn more biomedcentral.com/submissions 